\title{
Gesellschaft für Pädiatrische Onkologie und Hämatologie - Standortbestimmung
}

\author{
Society for Pediatric Oncology and Hematology - A Position Paper
}

Es war in der Vergangenheit guter Brauch in den „Ergebnisse der Pädiatrischen Onkologie“ über die Entwicklungen unserer Gesellschaft zu berichten. Mein Ausscheiden aus dem Amt des 1. Vorsitzenden gibt mir willkommene Gelegenheit diese Tradition fortzusetzen.

Ein wichtiges Ereignis der jüngeren Vergangenheit ist die Vereinigung von DAL und GPO zur GPOH. Die Gesellschaft für Pädiatrische Onkologie (GPO) bestand seit dem 7. März 1974. Zur besseren Bewältigung der zunehmend komplexen Aufgaben wurden am 29. 11. 1991 die satzungsgemäßen Ziele der bereits 1965 gegründeten „Deutschen Arbeitsgemeinschaft für Leukämieforschung und -behandlung im Kindesalter e.V." (DAL) in die der GPO aufgenommen und die Arbeitsgemeinschaft aufgelöst. Gleichzeitig wurden die Vereinsziele erweitert um die Förderung auch der nicht onkologischen Hämatologie und der Name geändert in „Gesellschaft für Pädiatrische Onkologie und Hämatologie" (4).

Im Zusammenhang mit der Wiedervereinigung Deutschlands haben wir uns über die problemlose Kooperation mit den Kollegen aus den neuen Bundesländern gefreut. Diese war möglich, weil wir uns bereits zu den Zeiten der DDR um einheitliche Behandlungsstrategien bemüht hatten und die Kollegen aus den neuen Bundesländern zunehmend in die Entscheidungsstrukturen eingebunden haben. Es bestand von Anfang an eine gegenseitige Wertschätzung, die dazu geführt hat, daß die Mehrzahl der Studien die neuen Bundesländer mit einbeziehen. Inzwischen konnte die Studienzentrale einer kooperativen Studie (Hodgkin-Studie) in einem der neuen Bundesländer angesiedelt werden.

Im Rahmen der Zusammenlegung von DAL und GPO und der thematischen Erweiterung um das Fach Hämatologie wurde auch eine Erweiterung des Vorstandes vorgenommen in der Absicht, alle wichtigen Teilgebiete, die im Rahmen interdisziplinärer Kooperation zur Lösung unserer Aufgaben beitragen, im Vorstand zu verankern, wie z.B. die Fächer Pathologie, Kinderchirurgie, Neurochirurgie, Radiotherapie, Hämatologie und Grundlagenforschung. Es wäre dabei zu überlegen, ob nicht die Berufung dieser Fachvertreter in den Beirat des Vorstandes eine praktikablere Form der Einbindung sein könnte. Gleichzeitig erfolgte auch die Integration der Psychosozialen Arbeitsgemeinschaft für Pädiatrische Onkologie (PSAPO) in die GPOH, wiederum mit Verankerung in unserer Gesellschaft durch einen Vorstandssitz, der grundsätzlich vom Vorsitzenden der PSAPO eingenommen werden soll.

Klin. Pädiatr. 207 (1995) 133-135

(C) 1995 F. Enke Verlag Stuttgart
Der Vorstand gab sich im Jahr 1990 erstmals eine $\boldsymbol{G e}$ schäftsordnung, welche Fragen der Mitgliedschaft, der Ausgaben, der Bildung von Strukturen für die Erfüllung der satzungsmäßigen Ziele, wie klinische Studiengruppen, Arbeitsgemeinschaften, zentrale Register u.a. regelt. Sie regelt desweiteren die Organisation der regelmäßigen Veranstaltungen, wie unserer wissenschaftlichen Halbjahrestagung, der Kind-Philipp-ForschungsTagung und der Bad-Nauheimer Studienleitertagung. Sie regelt die Herausgabe des Jahresheftes „Ergebnisse der Pädiatrischen Onkologie“, die Findung der Preisträger für den Förderpreis der GPOH sowie die Zusammenarbeit mit der DLFH.

Zur Bewältigung der stetig wachsenden Aufgaben (im Berichtsintervall stieg die Mitgliederzahl von 490 auf 655Mitglieder an) wurde 1992 beim Vorsitzenden ein $\mathbf{S e}$ kretariat eingerichtet und 1993 Frau PD Dr. Creutzig zur hauptamtlichen Geschäftsführerin der GPOH berufen. Dies ermöglicht ein professionelles Arbeiten unserer Gesellschaft und ist im Licht der vor uns liegenden schwierigen Zeiten eine gute Investition.

Die Mitwirkung der pädiatrischen Onkologen in der wissenschaftlichen Sektion der Deutschen Krebsgesellschaft erfolgt über die Arbeitsgemeinschaft für Pädiatrische Onkologie (AP0). Diese aus vereinsrechtlichen Gründen erforderliche Struktur hat nie eigenes Leben erlangt, weil schon vor ihrer Gründung die Pädiatrische Onkologie in DAL und GPO gegliedert und strukturiert war. Um jedoch eine wirkungsvolle Mitarbeit in der Deutschen Krebsgesellschaft zu sichern, beschlossen die Mitglieder der APO auf Anregung des GPOH-Vorsitzenden, grundsätzlich diesen als ihren Vorsitzenden anzusehen und auf regelhafte eigene Mitgliederversammlungen neben denen der GPOH zu verzichten.

Der Vorstand hat im zurückliegenden Zeitraum die wiederkehrenden Geschäfte, wie die wissenschaftlichen Halbjahrestagungen, die jährlichen Kind-Philipp-Forschungstagungen und Bad-Nauheimer Studienleitertagungen fortgeführt sowie auch die Herausgabe der Jahreshefte „Ergebnisse der Pädiatrischen Onkologie" betrieben.

Die GPOH-Tagungen wurden umstrukturiert. Es galt, die Hämatologie zeitlich zu integrieren. $\mathrm{Zu}$ diesem Zweck wurde der Beginn der Freitagssitzung um einige Stunden vorverlegt. Thematisch wurde nicht mehr in solide Tumoren und maligne Systemkrankheiten unterschieden, sondern in "klinischer Teil und klinische Forschung" einerseits und „kliniknahe Grundlagenforschung" andererseits. Die Tagung wurde darüberhinaus im Winter zu einer gemeinsamen Veranstaltung mit der Konferenz onkologischer Krankenschwestern (KOK) 
sowie der PSAPO gestaltet. Dieser Teil ist am Freitagvormittag der GPOH-Tagung vorangestellt. Gleichzeitig beschloß der Vorstand, nachdem das Angebot im Rahmen der Mitgliederumfragen mit großem Interesse aufgenommen wurde, einmal jährlich eine Fortbildungsveranstaltung durchzuführen. Diese soll dann jeweils im Sommer am Freitagvormittag der eigentlichen wissenschaftlichen GPOH-Tagung vorangestellt werden und wird erstmals im Sommer 1995 stattfinden. Die Abstracts unserer wissenschaftlichen Sitzungen werden nun veröffentlicht in der Monatsschrift für Kinderheilkunde. Um eine möglichst effektive Tagung zu haben, werden diese Abstracts unseren Tagungsteilnehmern bei Tagungsbeginn als Sonderdruck ausgehändigt, erstmals zur Wintertagung 1994.

Das Jahresheft „Ergebnisse der Pädiatrischen Onkolo-

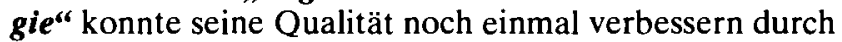
die Einschaltung von Gutachtern, welchen die jeweiligen Herausgeber die eingesandten Arbeiten zuschicken. Erstmals wurde dies 1992 so gehandhabt. Der Vorstand geht davon aus, daß diese Maßnahme erheblich zur Verbesserung des Ansehens unseres Jahresheftes beitragen wird.

Das vom GPOH-Vorstand angeregte und 1991 gestartete Modellprojekt des BMG ,Teilstationäre Versorgung krebskranker Kinder", an dem 10 klinische Einrichtungen partizipieren, läuft 1995 aus. Es hat sich gut entwikkelt und die Verhandlungen über eine Umsetzung in die Regelversorgung sind regional im vollen Gange und erscheinen aussichtsreich.

Der Vorstand hat jedoch nicht nur die traditionellen Geschäfte der Gesellschaft weitergeführt, sondern er hat sich um neue Strukturen bemüht sowie für neue Probleme nach Lösungen gesucht.

Er hat dafür gesorgt, daß die Zweitmalignome nunmehr systematisch über das Mainzer Kinderkrebsregister erfragt und erfaßt werden. Diese Form der Erfassung schien dem Vorstand notwendig, um die erforderliche Erfassungsdichte zu erreichen, auch schien es notwendig, die Möglichkeit nutzbar zu machen, den gesamten $\mathrm{Da}$ tenkörper des Mainzer Kinderkrebsregisters für eine wissenschaftliche Analyse der Zweitmalignome verfügbar zu haben. Damit wird die sehr verdienstvolle und auch erfolgreiche Arbeit von Herrn Gutjahr mit seiner Mitwirkung weitergeführt und institutionell abgesichert.

Der Vorstand stimmte der Gründung dreier neuer Arbeitsgemeinschaften zu, nämlich der AG für Pädiatrische Radio-Onkologie (APRO), der Arbeitsgemeinschaft Hyperthermie sowie der AG Knochenmarktransplantation. Der Vorstand seinerseits regte weitere Arbeitsgemeinschaften an, wie die Arbeitsgemeinschaft Spätfolgen sowie die AG Angewandte Informatik in der Pädiatrischen Onkologie.

Die Arbeitsgemeinschaft Spätfolgen unter Leitung von Herrn Prof. Beck hat wissenschaftliche Projekte z. B. für die Erfassung kardialer oder zentralnervöser Spätfolgen auf den Weg gebracht. Darüberhinaus hat sie in vielfältigen Tagungen für alle erdenklichen Spätfolgen Empfehlungen zu deren Erfassung erarbeitet. Eine Zusammen- fassung dieser Empfehlungen in praktikabler Form als Basis für ein einheitliches Vorgehen bei der Nachsorge erscheint an anderer Stelle in diesem Heft.

Die AG Angewandte Informatik in der Pädiatrischen Onkologie unter der Leitung von Herrn Prof. Michaelis hat sich in einem speziellen Arbeitskreis mit therapieunterstützenden Systemen befaßt und unter verschiedenen Möglichkeiten sich für die Weiterentwicklung des Systems CATIPO von Herrn Bachert, Heidelberg, entschieden. Diese wurde dankenswerterweise finanziell getragen von der Deutschen Leukämie-Forschungshilfe (DLFH). CATIPO steht unterdessen allen Kliniken zur Verfügung und bewährt sich zunehmend als ein wichtiges Instrument im Dienst der Arbeitserleichterung der Qualitätssicherung und auch bald für die Dokumentation. In einem weiteren Arbeitskreis unter der Federführung von Herrn Sauter wurde im sog. Basisdatensatz eine Systematik definierter Sachverhalte in der Pädiatrischen Onkologie als Grundlage für eine einheitliche Dokumentation erarbeitet (5). Er ist die Voraussetzung für eine weitere Entwicklung der elektronischen Datenverarbeitung in der Pädiatrischen Onkologie. Ein dritter Arbeitskreis unter der Federführung von Herrn Schilling befaßt sich mit Inhalten und Format der klinischen Dokumentation zur Erarbeitung der nächstnotwendigen Schritte: Dokumentation der klinischen Daten, Diagnoseverschlüsselung, Arztbriefschreibung, Weitergabe der für die Studienleitungen erforderlichen Daten etc.

Der Vorstand mußte sich auch mit Rechtsfragen im Rahmen unserer Studien befassen. Hier ging es einerseits um die Abgrenzung unserer „Therapieoptimierungsstudien" von klinischen Arzneimittelprüfungen, die dem AMG unterstehen. Hierzu ist im letzten Ergebnisheft ein Artikel von Frau Creutzig und mir erschienen (2), welcher die Probleme darstellt. Desweiteren ging und geht es darum, daß die Kosten für die im Rahmen der Studien eingesetzten Arzneimittel sowie die Kosten für die gesamte Behandlung von den Krankenkassen übernommen werden, wird doch das, was für uns wie eine Selbstverständlichkeit klingt, gegenwärtig sehr kontrovers diskutiert.

Außerdem befaßte sich der Vorstand mit Aspekten der Qualitätssicherung, die nach dem Sozialgesetzbuch 5 bindend vorgeschrieben ist. Hierzu wurde eine Analyse des Ist-Soll-Zustandes in der Pädiatrischen Onkologie vorgenommen und daraus ein Positionspapier erarbeitet, das der Qualitätssicherungskommission der Deutschen Gesellschaft für Kinderheilkunde weitergereicht wurde. Darüberhinaus wurde in Zusammenarbeit mit der Deutschen Krebsgesellschaft ein Symposium auf dem diesjährigen Krebskongreß zu diesem Thema veranstaltet, dessen Beiträge in einer Monographie veröffentlicht wurden (3). Als Teil der Maßnahmen zur Qualitätssicherung sind auch die Bemühungen des Vorstandes zu sehen, eine Fakultative Weiterbildung für pädiatrische Hämatologie und Onkologie zu etablieren.

Im wesentlichen in der Absicht, die Finanzierung des organisatorischen Aufwandes für die Therapieoptimierungsstudien auch für die Zukunft zu sichern, führte der Vorstand Gespräche mit der Deutschen Krebshilfe, der Deutschen Krebsgesellschaft und dem Verband der An- 
gestelltenkrankenkassen. Mit der Krebshilfe fanden mehrere Treffen statt, zuletzt am 31. 08. 1993 mit Vertretern der Vorstände von DKH, DLFH und GPOH. Dabei ergab sich, daß die Deutsche Krebshilfe grundsätzlich zu einer Fortsetzung der Förderung von Projekten in der Pädiatrischen Onkologie in dem bisherigen Finanzvolumen bereit ist. Die Therapieoptimierungsstudien allerdings werden, zumindest in großen Anteilen, als Maßnahmen der Qualitätssicherung angesehen, deren Kosten im Rahmen der Regelfinanzierung zu decken seien. Die Deutsche Krebshilfe zeigte sich interessiert, zwei bis drei molekulargenetisch ausgerichtete Einheiten schwerpunktmäßig zu fördern. Weitere Details hierzu wurden noch nicht abgestimmt.

In der Deutschen Krebsgesellschaft arbeiteten Vorstandsmitglieder in der Strategiekommission für Klinische Studien mit. Dort wurde eine Kommission Klinische Studien ins Leben gerufen, welche über die Vergabe von Fördermitteln entscheidet. Die in der pädiatrischen Onkologie vorherrschenden Therapieoptimierungsstudien werden voraussichtlich nicht von der Pharmaindustrie finanziert. Sie können weiter durch die Deutsche Krebshilfe finanziert werden. Hierfür wurde eine von Vertretern der Krebshilfe und Vertretern der Deutschen Krebsgesellschaft gebildete Leitkommission eingesetzt. Diese beurteilt die Förderwürdigkeit eines beantragten Projektes unter Hinzuziehung weiterer Gutachter. Seitens des Vorstandes wurden hierfür vier geeignete Mitglieder namhaft gemacht.

Mit Vertretern des $\boldsymbol{V D A} \boldsymbol{K}$ wurde ein erstes und ermutigendes Gespräch geführt über die Finanzierung von Therapieoptimierungsstudien unter dem Aspekt der Qualitätssicherung. Nach erfolgreichem Abschluß dieser Verhandlungen sind gegebenenfalls gleichartige Verhandlungen mit den RVO-Kassen erforderlich. Die Deutsche Krebsgesellschaft versucht ihrerseits in grundsätzlichen Verhandlungen mit den Krankenkassen eine Finanzierung der Therapieoptimierungsstudien zu erlangen.

Mit der $\boldsymbol{D L F H}$ wurde ein neues Kooperationspapier vereinbart, welches die finanzielle Stützung der Administration unserer Gesellschaft regelt und Grundsätze aufstellt über Ziele, Grenzen und Verfahrenswege bei der Förderung von Einzelprojekten wie von zentralen Serviceeinrichtungen. Der GPOH-Vorstand hat mitgewirkt bei der Einrichtung der Deutschen Kinderkrebsstiftung der DLFH, welche die Finanzierung der Förderung der pädiatrischen Onkologie langfristig sichern soll.

Der Vorstand hat darüberhinaus Aktivitäten entfaltet im Zusammenhang mit den Vorgängen um Katharina S., jenem leukämiekranken Kind, dessen Eltern die schulmedizinische Behandlung zugunsten einer alternativen Behandlung abgebrochen haben, ein Fall, der wegen seiner dramatischen Umstände hohes Interesse in der Öffentlichkeit fand. Hier wurde auf Betreiben des Vorstandes bei der Bundesärztekammer eine Expertenkommission eingerichtet. Diese Kommission hat eine Stellung- nahme erarbeitet, die im Deutschen Ärzteblatt publiziert wurde (1).

Der Vorstand hat auch geprüft, inwieweit eine von einer holländischen Fernsehproduktionsgesellschaft angebotene europäische Benefizveranstaltung "Thanks for Life" zugunsten der Pädiatrischen Onkologie realisierbar ist. Das Unternehmen konnte dann zunächst nicht weiter verfolgt werden, weil von den Nutznießern eine Vorfinanzierung verlangt wurde. Sowohl die DKH als auch die DLFH mußten eine solche Vorfinanzierung aus Spendengeldern ablehnen.

Insgesamt denke ich, hat der Vorstand gute Arbeit geleistet und vielfältige Entwicklungen eingeleitet. Aber auf den neuen Vorstand kommen zahlreiche neue Aufgaben und Probleme zu, wie zum Beispiel

- Aufbau eines elektronischen Datenaustauschs zwischen Kliniken, Studienzentralen und dem Mainzer Kinderkrebsregister

- Implementierung und Evaluation weiterer qualitätssichernder Maßnahmen

- Verhandlungen mit den Krankenkassen über die Finanzierung von Therapieoptimierungsstudien als qualitätssichernde Maßnahmen

- Mitwirkung an der Erarbeitung von Abteilungspflegesätzen und Fallpauschalen, wie sie das Gesundheitsstrukturgesetz vorschreiben

- die Realisierung einer fakultativen Weiterbildung, um nur einige der vordringlichen Aufgaben zu benennen.

Der Vorstand bedankt sich für das entgegengebrachte Vertrauen und wünscht dem neuen Vorstand Glück und Erfolg für seine Aufgabe.

\section{Literatur}

\begin{abstract}
Arbeitskreis des Wissenschaftlichen Beirats der Bundesärztekammer, Mitglieder: K. D. Bachmann, U. Creutzig, Ch. Fonatsch, G. JankaSchaub, H. Jürgens, B. Kornhuber, J. Michaelis, H. L. Schreiber, K. Winkler, G. Wolfslast, H.B. Wuermeling: Ethische und rechtliche Probleme bei der Behandlung bösartiger Erkrankungen bei Kindern und Jugendlichen. Dt. Ärzteblatt 91 (1994) B-2353-2357

2 Creutzig, U., K. Winkler: Empfehlungen für Studien zur Optimierung von Therapieschemata. Klin. Pädiatr. 206 (1994) 191-193

${ }^{3}$ Qualitätssicherung in der Onkologie. 1.1 Grundlagen und Definition, E. Enghofer und K. Winkler (Hrsg.), W. Zuckschwerdt Verlag (1995) 4 Satzung der Gesellschaft für Pädiatrische Onkologie und Hämatologie e. V. Gemeinnütziger Verein Frankfurt. Klin. Pädiatr. 206 (1994) 194195

5 Sauter, S., P. Kaatsch, U. Creutzig, J. Michaelis, für die Arbeitsgemeinschaft Angewandte Informatik der GPOH: Erstellung eines einheitlichen Basisdatensatzes für den Bereich der pädiatrischen Onkologie. Klin. Pädiatr. 206 (1994) 306-312
\end{abstract}

Prof. Dr. Kurt Winkler

Vorsitzender der Gesellschaft für

Pädiatrische Onkologie und Hämatologie

Universitäts-Kinderklinik

Martinistr. 52

D-20246 Hamburg-Eppendorf 